\title{
Kinderkrankheiten und Kinderheilkunde
}

\section{J. Oehme}

Honorar Professor an der Univ. Marburg/Lahn
Kinderkrankheiten gibt es, seitdem es Kinder gibt. Vorwiegend verstand man darunter die Krankheiten junger Kinder, insbesondere die Infektionskrankheiten wie Pocken, Masern u.a. in der irrigen Annahme, daß diese allein im Kindesalter vorkommen. Später erkannte man, daß jede beim Kind vorkommende Erkrankung ihren eigenen Charakter hat und dehnte den Begriff Kinderkrankheiten auf alle beim Kind vorkommenden Krankheiten aus ( $R$. För ster 1862).

Schon die alten Griechen kannten die Bezeichnung Kinderkrankheiten; eine Ubersicht gab Hippokrates. Der persische Arzt Rhazes veröffentlichte im 10. Jahrhundert die erste namentlich bekannte Schrift, die sich ausschließlich mit Kinderkrankheiten befaßte. Einen frühen Traktat aus Handschriften des 12 . bis 16 . Jahrhunderts stellte $K$. Sudhoff unter dem Titel „Practica Puerorum“ (Kinderpraxis) zusammen (zit. nach Peiper). Nach Entdeckung der Buchdruckerkunst erschienen in kürzester Zeit 3 gedruckte Bücher über Kinderkrankheiten. Das erste stammt von P. Bagellardus (gest. 1494); dann folgt das erste gedruckte Buch in deutscher Sprache von B. Metlinger (gest. 1492) mit dem Titel „Ein Regiment der jungen Kinder" (1473) und schließlich stellte Cornelius Roelans von Mecheln (1485) das ganze Wissen des 15. Jahrhunderts über Kinderkrankheiten zusammen. Das erste Buch über Kinderkrankheiten in englischer Sprache gab Thomas Phaer (gest. $1560)$ heraus, das erste Werk in französischer Sprache (5 Bücher) $S$. de Vallambert (1565). In der Folgezeit erschienen zahlreiche Schriften (Lit. bei Peiper), aber erst im 18. Jahrhundert erscheinen selbständige Bücher, die geeignet sind, die Grundlagen unseres Faches zu legen. Besonders zu erwähnen sind die Monographien des Schweden Nils Rosén von Rosenstein (1764) und des Engländers $M$. Underwood (1784). Von Bokay bezeichnete den Schweden als den ersten wirklichen Vertreter der Kinderheilkunde und L.T. Morton vertritt die Meinung, daß Underwood den Grundstein der modernen Pädiatrie gelegt hat (vgl. Ubersicht bei Oehme). Die Grundlagen der wissenschaftlichen Padiatrie entstehen im 19. Jahrhundert zunächst durch französische Ärzte (Ch Billard, E. Bouchut sowie $R$. Barthez und $F$. Rilliet), später erlebte die Pädiatrie im deutschsprachigen Raum einen ersten Höhepunkt. Der Internist $C$. Gerhardt gab mit zahlreichen pädiatrischen Fachvertretern in den Jahren 1877 bis 1896 das erste Handbuch der Kinderkrankheiten heraus.

\section{Kinderheilkunde (Pädiatrie)}

(Paediatrik - Kinderkunde - Pädologie)

Die Bezeichnung Pädiatrie taucht erstmalig 1722 auf in dem Titel des lateinisch geschriebenen Buches „Paedojatreja practica“ des Baseler Arztes Theodor Zwinger III. Darin werden zahlreiche Krankheiten beschrieben einschließlich solcher chirurgischer, dermatologischer und psychosomatischer Art; sogar Krankheiten der Augen und der Ohren bei Kindern gehören dazu. Soweit ich sehe, wurde der Ausdruck Kinderheilkunde erst von dem Paderborner Arzt P.A. Pieper in seinem Buch „Die Kinderpraxis" (1831) verwendet. Darin berichtete Pieper über seine Erfahrungen an den Kinderkrankenhäusern in Paris und erwähnt, daß man damals nach Frankreich gehen mußte, um eine „Ausbildung in Kinderheilkunst" zu erwerben. Im ,Brockhaus“ (Lexikon 1866) sucht man vergeblich das Stichwort Kinderheilkunde.

Lange Zeit mied man den Ausdruck Kinderheilkunde, der einen standespolitischen Unterton enthielt. Erst Ende des 19. Jahrhunderts wurde diese Bezeichnung zur Durchsetzung des eigenen Faches vielfach gebraucht (s.u.). Dieser feine terminologische Unterschied zwischen Kinderheilkunde und Kinderkrankheiten wird in neuerem Schrifttum wenig beachtet; vielfach werden diese Begriffe sogar wahllos gebraucht.

Später taucht der Ausdruck Pädiatrik (vgl. engl. paediatrics und auch pediatrics) auf. F.L. Meißner stellte 1850 die etwa 7000 Titel von Arbeiten über Kinderkrankheiten in seinem Buch „Grundlage der Literatur der Pädiatrik“ zusammen. Auch die erste Versammlung der „Kinderärzte“ im Rahmen der deutschen Naturforscher und Ärzte nannte sich Sektion für Pädiatrik (1868). In Österreich hat Mauthner 1858 beantragt, Pädiatrik als Prüfungsfach im Rigorosum aufzunehmen. 1899 wurde in Österreich die Kinderheilkunde zum Prüfungsfach erhoben, in Deutschland erst 1918.

Es ist deshalb kein Zufall, daß die erste Zeitschrift, die im Titel den Terminus „Kinderheilkunde“ enthielt, in Osterreich herausgegeben wurde. Der Stettiner Kinderarzt $A$. Steffen, der die pädiatrische Sektion der Naturforscherversammlung gegrundet hatte, wird 1868 Mitherausgeber des „Jahrbuches für Kinderheilkunde und physische Erziehung“, das 10 Jahre zuvor erstmalig in Wien erschien und nun einzugehen drohte. Wie allgemein bekannt, gründete $A$. Steffen gegen heftigen Widerstand der Internisten dann 1883 
die Gesellschaft für Kinderheilkunde, deren 100jähriges Jubiläum wir voriges Jahr feierten. In rascher Reihenfolge erscheinen jetzt kinderärztliche Zeitschriften, die im Titel das standesbewußte Wort „Kinderheilkunde“ tragen. Schon 1880 war das Archiv für Kinderheilkunde erschienen (jetzt: „Klinische Pädiatrie“), 1902 folgte die Monatsschrift; 1910 die Zeitschrift (jetzt: European Journal of Pediatrics) und 1911 das Zentralblatt für Kinderheilkunde. Auch das von den Pädiatern M.v. Pfaundler u. A. Schloßmann herausgegebene mehrbändige Handbuch (1906) heißt jetzt im Gegensatz zu dem vom Internisten Gerhardt herausgegebenen „Handbuch der Kinderheilkunde". Inzwischen war es auch durch die Unterstützung des preußischen Ministerialdirektors F. Althoff gelungen, in Berlin einen ordentlichen Lehrstuhl für Kinderheilkunde zu errichten, den 1894 Otto Heubner einnahm.

1918 wurde nach wiederholten Schreiben und Eingaben Gesellschaft für Kinderheilkunde erreicht, daß Pädiatrie Prüfungsfach wurde. Uber die weitere Entwicklung habe ich an anderer Stelle ausfuihrlich berichtet (Therapiewoche 84).

\section{Aufgabenbereich}

Schon frühzeitig tauchte die Frage nach dem Aufgabenbereich der Kinderheilkunde auf. Während $A$. Czerny der Meinung war, daß Kinderheilkunde Innere Medizin begrenzt vom Tage der Geburt bis zur Pubertät sei, wurde schon von Zwinger (s.o.) und später von der PfaundlerSchule die Meinung vertreten, daß alle Krankheiten beim Kind zunächst vom Kinderarzt angesehen bzw. behandelt werden sollten. Auch A. Steffen hat im Gegensatz zu der Auffassung von Czerny betont, daß Kliniken sowohl für interne wie chirurgisch kranke Kinder in einem Haus sein sollten. Der große Sozialpädiater $A$. Schloßmann ging in seinem Referat auf der Tagung unserer Gesellschaft in Hamburg (1928) noch weiter, wenn er ausrief: „Das Kinderkrankenhaus ist tot, es lebt und wird leben das Haus für die kranken Kinder. Kinder haben auf Augenkliniken, auf Ohrenkliniken, auf sog. chirurgischen Tuberkulosestationen oder gar auf Hautkliniken nichts zu suchen, sie gehören alle miteinander auf die Kinderabteilung." (Schloßmann). Schloßmann war es auch, der den Präventivauftrag des Kinderarztes in der Bezeichnung Kinderheilkunde nicht genügend berücksichtigt fand. Er schlug deshalb wie auch. später Hamburger die Bezeichnung Kinderkunde vor, die sich allerdings nicht einbürgerte. Der Ausdruck Pädologie schließlich, ursprünglich für alle Wissenschaften, die sich mit dem Kinde befassen, geprägt, wurde von Linneweh auf die physiologischen Besonderheiten des Kindesalters reduziert.

Einigkeit besteht darüber, daß neben der Therapie der Prophylaxe gerade bei Kindern eine erhöhte Bedeutung zukommt. Damit befinden sich die Kinderärzte in guter Ubereinstimmung mit den medizinischen Anschauungen Ende des 18. Jahrhunderts (vgl. auch den Titel „Jahrbuch für Kinderheilkunde und physische Erziehung "). Zahlreiche populärmedizinische Schriften trugen Titel wie „Allgemeine Regeln, seine Gesundheit zu erhalten" (S.A. Tissot) oder "Die Kunst, das menschliche Leben zu verlängern" $(C . W$. Hufeland). Es ist an der Zeit, daß die „Diaita“, die heutzutage auf Diät reduziert wurde, wieder in ihrer ganzen Bedeutung erkannt, und der Kinderarzt sich den Fragen der Lebensordnung und der Gesundheitsplanung vermehrt zuwendet; sonst besteht die Gefahr, daß diese Fragen ohne ihn behandelt werden (Wollinger). Dies gilt auch für die Erziehung. Czerny hat dies in seinem Buch „Der Arzt als Erzieher des Kindes" 1908 klar ausgesprochen.

\section{Literatur}

zusammenfassend bei:

Peiper, A.: Chronik der Kinderheilkunde. 4. Auf lage, Leipzig 1965 Schweier, P., E. Seidler: Lebendige Pädiatrie. Marseille Verlag, München 1983

ferner:

Bockay, J.v.: Die Geschichte der Kinderheilkundè. Berlin 1922 Czerny, A.: Pädiatrie meiner Zeit. Berlin 1939

Förster, R.: Vortrag in der Gesellschaft für Natur- und Heilkunde zu Dresden 1862

Linneweh, F.: Die Fortschritte der Pädologie. 2. Auflage, Berlin 1968

Morton, L.T.: A Medical Bibliographie, 4. Auflage, London 1983

Oehme, J.: Therapiewoche 34 (1984) 1532 und Pädiatrie im 18. Jahrhundert, Documenta Pädiatrica Band 12, Lübeck 1984

Seidler, E.: der kinderarzt 18, 5

und Heidelberger Jahrbücher 10, 83 (1966

Wollinger, $R .:$ der kinderarzt $14,15,37$ (1983) 